\title{
因HAD
}

DOI: http://doi.org/10.22585/hospdomic.v2i3.50

\section{Análisis bibliométrico y temático de la producción científica existente en la base de datos bibliográfica MEDLINE sobre medicamentos peligrosos en las Unidades de Hospitalización a Domicilio}

Bibliometric and thematic analysis of the scientific literature on hazardous drugs in home care services based in the hospital, in the MEDLINE bibliographic database

Mari A. Bernabeu-Martínez', Javier Sanz Valero²

1. Hospital General Universitario de Alicante, Alicante, España.

2. Universidad Miguel Hernández de Elche, Departamento de Salud Pública, Historia de la Ciencia y Ginecología, Campus Sant Joan d'Alacant, España.

Correspondencia/Correspondence

Mari A. Bernabeu-Martínez

Universidad Miguel Hernández de Elche,

Departamento de Salud Pública, Historia de

la Ciencia y Ginecología, Campus Sant Joan

d'Alacant, España.

bernabeu_marmar@gva.es

Recibido/Received

04.07.2018

Aceptado/Accepted

07.07.2018
Conflicto de Intereses/Competing interest

Los autores declaran la inexistencia de cualquier tipo de conflicto de interés.

CÓMO CITAR ESTE TRABAJO | HOW TO CITE THIS PAPER

Bernabeu-Martínez M A. Sanz Valero J. Análisis bibliométrico y temático de la producción científica existente en la base de datos bibliográfica MEDLINE sobre medicamentos peligrosos en las Unidades de Hospitalización a Domicilio. Hosp Domic. 2018;2(3):101-15. 


\section{RESUMEN}

Objetivo: Analizar y caracterizar, mediante técnica bibliométrica, la documentación científica relacionada con los medicamentos peligrosos en las Unidades de Hospitalización a Domicilio indizada en la base de datos bibliográfica MEDLINE.

Método: Estudio descriptivo transversal. Los datos se obtuvieron de la base de datos MEDLINE, a través de PubMed, interrogando los términos a estudio en los campos de descriptores, título y resumen; fecha de búsqueda mayo 2018.

Resultados: Se analizaron 74 referencias. El número de originales fue de 32 (43,2\%), identificando 56 instituciones, con Índice de Cooperación de 1,4 \pm 0,1 autores/artículo. El idioma predominante fue el japonés con 36 (48,6\%) artículos. La obsolescencia, según el Índice de Burton-Kebler fue de 15 años y el Índice de Price del 9,5\%. El núcleo de Bradford lo constituyó 1 revista. El descriptor más utilizado fue Home Care Services, Hospital-Based ( $n=52 ; 70,3 \%)$ y el área temática más representada Health Care Category, $(n=134 ; 34,4 \%)$

Conclusiones: Los estudios sobre medicamentos peligrosos en el hospital a domicilio son un área temática en decrecimiento, con una gran fragmentación y poco uniforme, sin grandes grupos de referencia ni una base sólida desde la que se puedan continuar estudios posteriores. El japonés es el idioma mayoritario. Los descriptores y las áreas temáticas son acordes al área estudiada, aunque sería conveniente, dada la creciente sensibilización con la manipulación de los MP, el desarrollo de un descriptor $\mathrm{MeSH}$ específico.

Palabras clave: Agentes Antineoplásicos; Sustancias Peligrosas; Agentes Citostáticos; Servicios de atención a domicilio provisto por hospital; Acceso a la Información; Bibliometría; Indicadores Bibliométricos; Descriptores de Ciencias de la Salud.

\section{ABSTRACT}

Objective: Analyze and characterize, through bibliometric technique, the scientific documentation related to hazardous drugs in home care services based in the hospital, indexed in the MEDLINE bibliographic database

Methods: Cross-sectional descriptive study. The data was obtained from the MEDLINE database, through PubMed, by interrogating the study terms in the descriptor, title and summary fields. Search date was May 2018.

Results: A total of 74 references were analyzed. The number of originals was 32 (43.2\%), identifying 56 institutions, with a Cooperation Index of $1.4 \pm 0.1$ authors / article. The predominant language was Japanese with 36 (48.6\%) articles. The obsolescence, according to the Burton-Kebler Index, was 15 years and the Price Index was $9.5 \%$. The core of Bradford included 1 journal. The most widely used descriptor was "Home Care Services, Hospital-Based" on 52 (70.3\%) occasions and the most represented theme area Health Care Category, 134 (34.4\%) times.

Conclusions: The scientific production on hazardous drugs in home care services based in the hospital is a declining and not uniform thematic area, with a great fragmentation, without large reference groups or a solid base from which further studies can be continued. Japanese is the majority language. The descriptors and the thematic areas are in accordance with the studied area, although it would be convenient, given the increasing awareness with handling hazardous drugs, the development of a specific MeSH descriptor, indexed in the MEDLINE database. The thematic classification fully corresponds to the subject matter investigated.

Keywords: Antineoplastic Agents; Hazardous Substances; Cytostatic Agents Home Care Services, Hospital-Based; Access to information; Bibliometrics; Bibliometric Indicators; Medical Subject Headings. 


\section{INTRODUCCIÓN}

La Hospitalización a Domicilio (HAD) es un modelo asistencial eficaz, seguro y satisfactorio para los pacientes y familiares, cuya función primordial es brindar al enfermo en su domicilio cuidados médicos y de enfermería en igual cantidad y calidad que en el hospital (1).

La necesidad de descongestionar los hospitales, así como el progresivo proceso de "ambulatorización" de la gestión sanitaria, han impulsado en los últimos 70 años el traslado de los cuidados sanitarios desde el hospital al domicilio del paciente a través de las Unidades de Hospitalización a Domicilio (HAD) (2). Así mismo, la generalización de las tecnologías de la información y la telemedicina, así como la disposición de nuevas herramientas, como la miniaturización de aparatos, han favorecido la asunción por parte de la HAD de enfermos que requieren una asistencia clínica de alta complejidad (3). Esta asistencia está estructurada en un marco de seguridad para el paciente, pero también para el profesional sanitario, sin olvidar a los familiares y cuidadores.

En este sentido, la administración de ciertos medicamentos peligrosos en el domicilio del paciente, como es el caso de fármacos antineoplásicos, práctica ampliamente extendida en las unidades de HAD de todo el mundo, exige que la manipulación de éstos se haga en base a las más estrictas normas de seguridad, con el fin de evitar los riesgos asociados a la exposición, tanto para el personal sanitario como para los familiares y cuidadores. Ya en 1988, The Joint Commission estableció estándares relacionados con la manipulación segura de sustancias peligrosas en el domicilio del paciente (4). No obstante, una revisión sistemática sobre guías de manipulación de medicamentos peligrosos (MP) dejó patente que tan solo una quinta parte de los documentos seleccionados abordaban el ámbito domiciliario (5).

La seguridad de los profesionales sanitarios debe convertirse en un estándar de práctica, donde el ámbito asistencial no sea una diferencia, más aún cuando una mala praxis en el domicilio del paciente puede suponer un riesgo añadido para los familiares que con él conviven.

En consecuencia, dada la creciente preocupación y sensibilización acerca del peligro que supone la manipulación de los MP en el entorno domiciliario, resulta útil conocer la progresión de su investigación y del conocimiento creado. El estudio de la producción científica mediante análisis bibliométrico permite examinar a través del cálculo de una serie de indicadores, determinados aspectos relevantes sobre una temática científica concreta, como por ejemplo en qué revistas/ plataformas se publica más, qué instituciones tienen mayor relevancia en el campo referido, o qué publicaciones son más visibles frente al ruido generado por la competitividad del mercado laboral $(6,7)$, qué grupos de investigación son los más destacados, además de medir la obsolescencia y la dispersión de las publicaciones.

No obstante, el cálculo de indicadores no puede limitarse a la aportación de datos estadísticos, sin más y por separado, sino que deben estar relacionados para que puedan aportar una explicación sólida sobre la actividad científica que se esté considerando (8). Por tanto, para relacionar los indicadores con un área de conocimiento específica es necesario encajar el cálculo métrico con el análisis temático.

Teniendo en cuenta lo anteriormente expuesto, el objetivo de este trabajo fue, analizar y caracterizar, mediante técnica bibliométrica, la documentación científica relacionada con la manipulación de MP en las unidades de HAD, indizada en la base de datos bibliográfica MEDLINE. 


\section{MATERIAL Y MÉTODO}

\section{Diseño}

Estudio descriptivo trasversal de la producción científica sobre la manipulación de MP en HAD.

\section{Fuente de obtención de los datos}

Se obtuvieron de la consulta directa y acceso, vía Internet, a la documentación científica existente en la base de datos MEDLINE, vía PubMed.

\section{Unidad de análisis}

Los artículos sobre MP en HAD indizados en la base de datos bibliográfica indicada.

\section{Búsqueda bibliográfica}

Para construir la ecuación de búsqueda se consultó el Medical Subject Headings (MeSH), Thesaurus desarrollado por la U.S. National Library of Medicine.

La ecuación de búsqueda empleada se desarrolló mediante la intersección booleana de dos ecuaciones [ecuación 1 AND ecuación 2]:

\section{Ecuación 1:}

("Antineoplastic Agents"[Mesh] OR "Antineoplastic Agents"[Title/Abstract] OR "Antineoplastic Drugs"[Title/Abstract] OR "Antineoplastics"[Title/Abstract] OR "Chemotherapeutic Anticancer Drug"[Title/Abstract] OR "Antitumor Drugs"[Title/Abstract] OR "Cancer Chemotherapy Agents"[Title/ Abstract] OR "Cancer Chemotherapy Drugs"[Title/Abstract] OR "Chemotherapeutic Anticancer Agents"[Title/Abstract] OR "Anticancer Agents"[Title/Abstract] OR "Antitumor Agents"[Title/Abstract] OR "Hazardous Substances"[Mesh] OR "Hazardous Substances"[Title/Abstract] OR "Hazardous Materials"[Title/Abstract] OR "Hazardous Chemicals"[Title/Abstract] OR "Environmental Toxic Substances"[Title/Abstract] OR "Toxic Environmental Substances"[Title/Abstract] OR "Biohazards"[Title/Abstract] OR "Cytostatic Agents"[Mesh] OR "Cytostatic Agents"[Title/Abstract] OR "Cytostatics"[Title/Abstract] OR "Cytostatic Drugs"[Title/Abstract] OR "Hazardous Drugs"[Title/ Abstract] OR "Chemotherapy" [Title/Abstract] OR "Chemotherapeutic Agents" [Title/Abstract] OR "Chemotherapeutic Drugs"[Title/Abstract] OR "Cytotoxic Drugs"[Title/Abstract] OR "Cytotoxics"[Title/ Abstract] OR "Antineoplastic medications"[Title/Abstract] OR "Anticancer Drugs"[Title/Abstract] OR "Highly potent drugs"[Title/Abstract])

\section{Ecuación 2:}

("Home Care Services, Hospital-Based"[Mesh] OR "Hospital-Based Home Care"[Title/Abstract] OR "Hospital Based Home Care"[Title/Abstract] OR "Hospital Home Care Services"[Title/Abstract] OR "Hospital-Based Home Care Services"[Title/Abstract] OR "Hospital Based Home Care Services"[Title/Abstract] OR "Home Hospitalization"[Title/Abstract] OR "Hospital at Home"[Title/Abs- 
tract] OR "Hospital-at-Home"[Title/Abstract] OR "Hospital Home Care"[Title/Abstract] OR "Hospital at Home Care"[Title/Abstract] OR "Hospital in the Home"[Title/Abstract]).

La búsqueda final se realizó en mayo de 2018.

\section{Indicadores a estudio}

- Producción científica, calculada según número de artículos indizados.

- Tipología documental y número de artículos citables (suma de artículos de investigación original y de revisión).

- Índice de productividad (logaritmo del número de trabajos originales publicados).

- Edad: 2018 menos año de publicación del artículo.

- Semiperiodo de Burton-Kebler (Mediana de la distribución del conjunto de las referencias ordenadas por su antigüedad).

- Índice de Price (porcentaje de referencias con edad menor de 5 años).

- Idioma de publicación del artículo.

- Distribución geográfica de procedencia de los artículos.

- Índice de colaboración institucional: Número de instituciones que figuran como firmantes del documento.

- Filiación institucional del primer firmante e Índice de Lotka. Las instituciones se agruparon en tres niveles de rendimiento: pequeños productores (Índice de transitoriedad = un único trabajo), medianos productores (entre 2 y 9 trabajos) y grandes productores (10 o más trabajos).

- Número de autores por artículo e índice de colaboración (cociente entre el número de firmas y el número de trabajos).

- Revista donde se publica el artículo.

- Impacto de las publicaciones según el Journal Citation Report Science Edition Database (JCR) y del SCImago Journal Rank (SJR).

- Dispersión: núcleo principal de Bradford (conjunto de revistas de mayor pertinencia para un área del conocimiento).

- Enlace: Existencia de enlace al documento desde PubMed.

- Acceso: Posibilidad de acceder al texto completo del artículo -gratuito o mediante pago- a través de del enlace existente en PubMed.

- Indización mayor: Descriptores (MeSH), como Major Topic, que representan el contenido principal de cada artículo.

- Área temática: clasificación de los artículos según las 16 grandes áreas temáticas del thesaurus MeSH al primer nivel.

\section{Análisis de los datos}

Las variables cualitativas se describieron por su frecuencia y relativa (porcentaje), las cuantitativas mediante su Media y Desviación Estándar, con Intervalos de Confianza del 95\%; representando las más relevantes mediante la utilización de tablas y gráficos. Se utilizó la Mediana, como medida de tendencia central, calculándose en ocasiones, el Máximo. Para conocer la evolución de algunos indicadores se segmentó el período de estudio en dos etapas, tomando como punto de corte el índice de Burton-Kebler (la Mediana): $1^{\mathrm{a}}$ época: de 1994 a 2003 y $2^{\mathrm{a}}$ época: de 2004 a 2018. El crecimiento de la producción científica se examinó mediante el análisis de regresión. La existencia de asociación entre variables cualitativas se analizó mediante la prueba de chi-cuadrado de Pear- 
son. Para comprobar la significación en la diferencia de medias para muestras independientes se utilizó la prueba t de Student. El nivel de significación utilizado en todos los contrastes de hipótesis fue $a \leq 0,05$.

Para el almacenamiento de datos y análisis estadístico se utilizó el programa Statistical Package for the Social Sciences (IBM-SPSS), versión 22 para Windows.

El control de la calidad de la información se efectuó mediante la doble entrada de los datos, corrigiendo las inconsistencias mediante la consulta con los datos originales.

\section{RESULTADOS}

\section{Producción científica}

La búsqueda efectuada en la base de datos MEDLINE dio un total de 74 referencias. El primer artículo indizado en la base de datos data del año 1994, publicado en la revista Gan to kagaku ryoho (Cancer \& chemotherapy) (9), siendo el 2006 el año de mayor producción sobre la temática a estudio, con 10 artículos, un $13,5 \%$ del total.

Al estudiar la relación entre el número de publicaciones por año se comprobó que el modelo de regresión con un mayor ajuste fue el exponencial decreciente, siendo el coeficiente de determinación $\mathrm{R} 2$ = 0,3762; ver figura 1 .

Figura 1. Evolución anual de la producción científica sobre MP en HAD, recuperada de MEDLINE en mayo de 2018, y ajuste al modelo exponencial

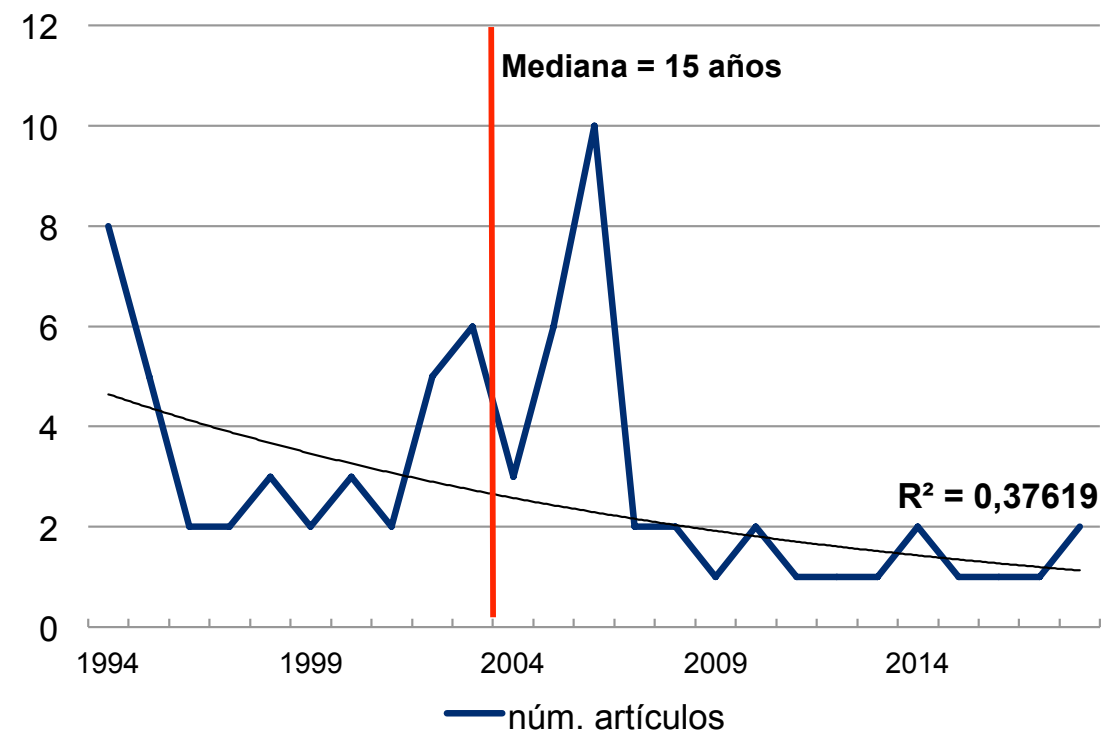




\section{Obsolescencia/actualidad de la producción científica}

La edad media de los documentos analizados fue de 14,7 \pm 0,75 años, con mediana de 15 años (Índice de Burton-Kebler) y máximo de 24 años; ver figura 1. El Índice de Price obtenido fue del $9,5 \%$.

\section{Tipología documental}

La tipología documental más frecuente fue el artículo original con 32 documentos (43,2\%), siendo el índice de productividad de 1,5. El número revisiones fue de 7 (9,5\%) y el de ensayos clínicos de $18(24,3 \%)$.

No se observaron diferencias significativas, relacionadas con la tipología documental, entre las dos épocas a estudio (chi cuadrado de Pearson $=9,4 ; g l=6 ; p=0,153$ ).

La frecuencia y porcentaje de los artículos citables (originales + revisiones), computables para el cálculo del impacto, fue de 57 (77,0\%).

\section{Procedencia geográfica y filiación institucional}

La distribución geográfica, de los autores que aparecen como primer firmante, fue preferentemente japonesa, responsable de la mitad de la producción: 37 trabajos (50\%). Le siguieron Francia, con 13 artículos (17,6\%) y en menor proporción diferentes países anglosajones: Australia 6 documentos $(8,1 \%)$, Canadá $3(4,1 \%)$, EE.UU $3(4,1 \%)$ y Reino Unido 3 trabajos $(4,1 \%)$. Los documentos con filiación española fueron $3(4,1 \%)$.

Tabla 1. Distribución por países de los trabajos indizados en MEDLINE sobre MP en HADa , ordenados según época de estudio

\begin{tabular}{|c|c|c|c|c|}
\hline \multirow[t]{2}{*}{ País } & \multicolumn{2}{|c|}{$\begin{array}{l}1^{a} \text { época } \\
1994 \text { a } 2003\end{array}$} & \multicolumn{2}{|c|}{ 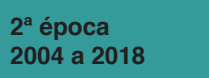 } \\
\hline & num & $\%$ & num & $\%$ \\
\hline Japón & 25 & 33,8 & 12 & 16,2 \\
\hline Francia & 4 & 5,4 & 9 & 12,2 \\
\hline Australia & 5 & 6,8 & 1 & 1,4 \\
\hline Canadá & 0 & 0,0 & 3 & 4,1 \\
\hline España & 1 & 1,4 & 2 & 2,7 \\
\hline EE.UU. & 0 & 0,0 & 3 & 4,1 \\
\hline Reino Unido & 1 & 1,4 & 2 & 2,7 \\
\hline
\end{tabular}


Los países con 3 o más publicaciones, ordenados según época, pueden consultarse en la tabla 1, no encontrándose diferencias significativas entre las dos épocas a estudio (chi cuadrado de Pearson $=17,8 ; \mathrm{gl}=10 ; \mathrm{p}=0,059$ ).

No se observó colaboración entre países en ninguno de los trabajos analizados.

Se identificaron trabajos publicados de 56 instituciones, con una Mediana de 1 centro por artículo y Máximo de 7. La media del número de instituciones por artículo -índice de colaboración institucional- fue de 1,4 $\pm 0,1$. No se observaron diferencias en las medias del Índice de colaboración institucional entre las dos épocas estudiadas ( 1,2 versus 1,7 ; de Student $=-2,0 ; \mathrm{gl}=41,9 ; \mathrm{p}=$ 0,058). Un total de $13(17,6 \%)$ estudios fueron realizados en colaboración entre 2 o más centros.

La filiación de los documentos analizados se clasificó, extrapolando el Índice de Lotka, en tres niveles de rendimiento: pequeños productores, o Índice de Transitoriedad (un único trabajo), donde encontramos 46 centros (82,1\%); medianos productores (entre 2 y 9 trabajos) con 10 centros $(17,9 \%)$; y grandes productores (10 o más trabajos) donde no se localizó ningún centro. En 1 artículo $(1,8 \%)$ no figuraba filiación.

\section{Idioma de publicación}

Los documentos revisados estaban predominantemente escritos en japonés, en 36 ocasiones (48,6\%), seguidos del inglés en 27 artículos (36,5\%), del francés en 7 trabajos $(9,5 \%)$, el español en 3 documentos $(4,1 \%)$ y el sueco en 1 único estudio $(1,4 \%)$. Se observaron diferencias significativas, en relación al idioma, entre las dos épocas a estudio (chi cuadrado de Pearson $=11,4 ; \mathrm{gl}=4 ; \mathrm{p}=$ 0,023) (Tabla 2).

Tabla 2. Distribución por idiomas de los trabajos indizados en MEDLINE sobre MP en HAD, ordenados según época de estudio

\begin{tabular}{|c|c|c|c|c|}
\hline \multirow[t]{2}{*}{ Idioma } & \multicolumn{2}{|c|}{$\begin{array}{l}1^{a} \text { época } \\
1994 \text { a } 2003\end{array}$} & \multicolumn{2}{|c|}{$\begin{array}{l}2^{\mathrm{a}} \text { época } \\
2004 \text { a } 2018\end{array}$} \\
\hline & num & $\%$ & num & $\%$ \\
\hline Japonés & 25 & 65,8 & 11 & 30,6 \\
\hline Inglés & 8 & 21,1 & 19 & 52,8 \\
\hline Francés & 3 & 7,9 & 4 & 11,1 \\
\hline Español & 1 & 2,6 & 3 & 4,1 \\
\hline Sueco & 1 & 2,6 & 0 & 0,0 \\
\hline
\end{tabular}

\section{Autoría}

Se contabilizaron un total de 420 firmantes, con un máximo de 11 autores observado en 4 artículos. La media de autores -índice de cooperación- fue de 5,68 $\pm 0,35$. La mediana fue de 5 autores por artículo y la moda de 4 autores. No se observaron diferencias significativas entre las medias 
de autores/artículo de las dos épocas a estudio: 5,3 versus 6,1 (t de Student $=-1,2 ; \mathrm{gl}=71,4 ; \mathrm{p}=$ $0,232)$; ver figura 2.

Figura 2. Número de autores/artículo de los trabajos indizados en MEDLINE sobre MP en HAD, ordenados según época de estudio

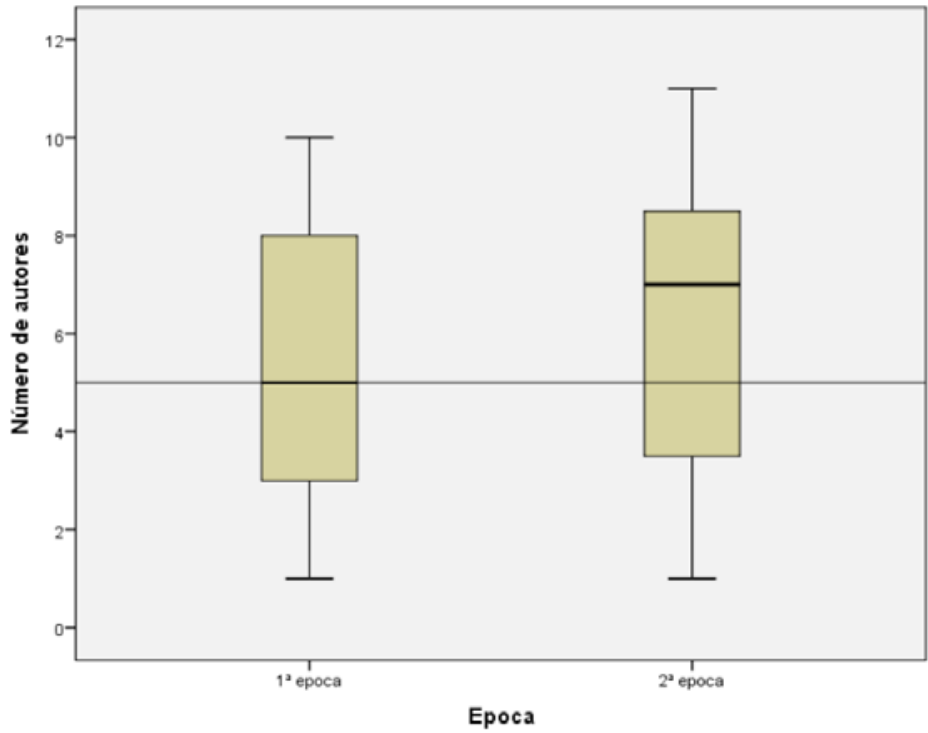

\section{Revistas, impacto y dispersión de la literatura científica}

Se recuperaron un total de 38 revistas que contenían los 74 artículos estudiados, de las que sólo 3 publicaron 2 o más artículos sobre el tema a estudio; ver tabla 3 que contiene la medición del impacto de estas publicaciones

Tabla 3.Revistas que han publicado 2 o más artículos, indizados en MEDLINE, sobre MP en HAD

\begin{tabular}{|c|c|c|c|c|c|c|}
\hline Abreviatura de la revista & $f_{0}$ & $\%$ & $\mathrm{FI}_{(\mathrm{JCR})}$ & $\mathbf{Q}_{(\mathrm{JCR})}$ & SJR & $\mathbf{Q}_{(\mathrm{SJR})}$ \\
\hline Gan To Kagaku Ryoho & 35 & 47,3 & --- & --- & --- & --- \\
\hline Aust J Adv Nurs & 2 & 2,7 & 0,511 & Q4 & 0,299 & Q2 \\
\hline Pediatr Blood Cancer & 2 & 2,7 & 2,646 & Q3 & 1,338 & Q1 \\
\hline \multicolumn{7}{|c|}{$\begin{array}{l}\text { fO = Número de artículos publicados; FI(JCR) = Factor de Impacto según la base de datos Journal Citation Report para 2017; } \\
\text { Q(JCR) = Cuartil según la mejor posición que ocupa la revista en cualquier categoría en la base de datos Journal Citation } \\
\text { Report; SJR = Indicador Scimago Journal Rank para } 2017 ; \text { Q(SJR) = Cuartil según la mejor posición que ocupa la revista en } \\
\text { cualquier categoría en la base de datos Scimago Journal \& Country Rank. }\end{array}$} \\
\hline
\end{tabular}


El estudio de la dispersión de la literatura científica recuperada, determina la concentración de una frecuencia similar de artículos en un número diferente de revistas; esta frecuencia de artículos se corresponde aproximadamente con los terciles de producción (33\% de documentos para cada una de las zonas). Así el núcleo principal (zona 1), con 1 revista (2,6\%) incluía 35 artículos (47,3\%); la zona 2 con 12 revistas $(31,6 \%)$ que contenía 14 artículos $(18,9 \%)$ y la zona 3 con 25 revistas (65,8\%) que englobaban 25 artículos (33,8\%); ver figura 3: gráfico de dispersión según Bradford.

Figura3. Dispersión de las revistas y los artículos sobre MP en HAD en la base de datos bibliográfica de MEDLINE (mayo 2018)

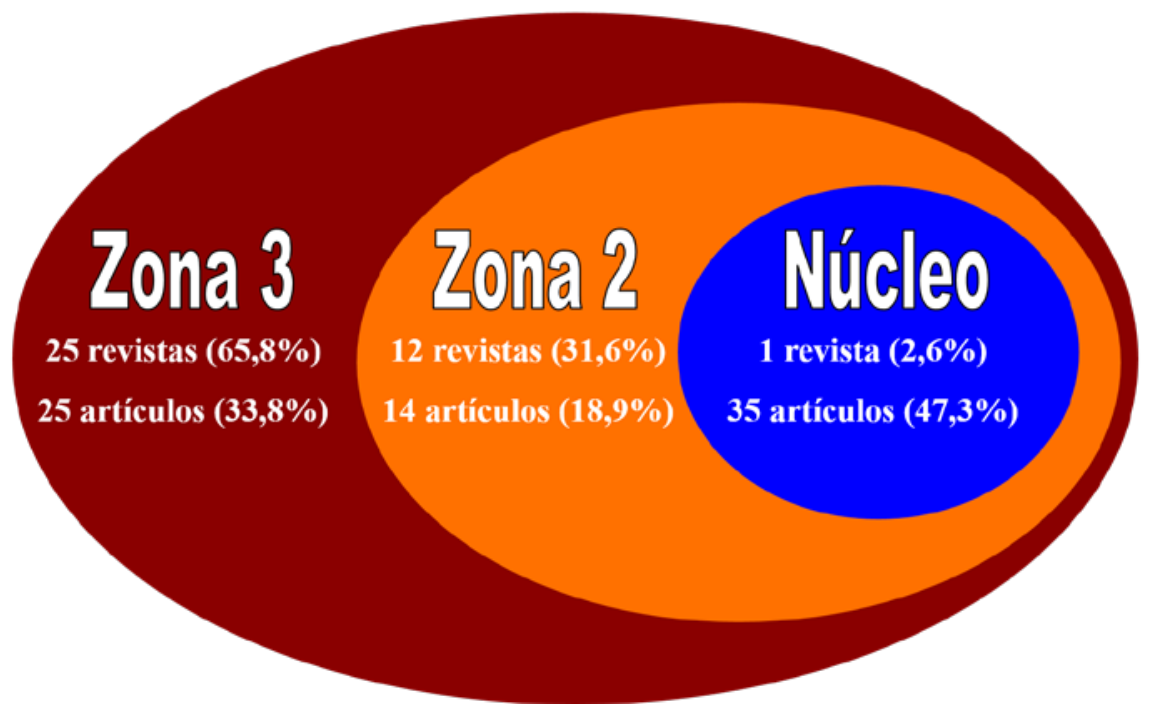

\section{Acceso al documento primario}

La existencia de enlace al texto completo desde MEDLINE se observó en 35 ocasiones (47,3\%), siendo en 14 ocasiones (18,9\%) de forma gratuita. Existieron diferencias significativas, a favor de la $2^{a}$ época, tanto en el enlace al documento ( $77,8 \%$ versus $18,4 \%$; chi cuadrado de Pearson $=26,1$; $\mathrm{gl}=1 ; \mathrm{p}<0,001)$, como a la consulta del texto de forma gratuita (30,6\% versus $7,9 \%$; chi cuadrado de Pearson = 6,2; $g l=1 ; p=0,013$ ).

Se constató que 27 documentos (36,5\%) estaban identificados mediante DOI, observando diferencias a favor de la $2^{\mathrm{a}}$ época $(61,1 \%$ versus $13,2 \%$; chi cuadrado de Pearson $=18,3 ; \mathrm{gl}=1 ; \mathrm{p}$ $<0,001)$.

\section{Clasificación temática de la producción científica}

Para conocer la pertinencia temática de la producción científica a estudio se comprobó que los 74 documentos seleccionados habían sido indizados mediante 118 diferentes Medical Subject Headings (MeSH), pertenecientes a 14 de las 16 áreas temáticas del Thesaurus de la U.S. National Library of Medicine. Los MeSH que fueron utilizados 10 o más veces pueden consultarse en la tabla 4. 
Tabla 4. Distribución de los Medical Subject Headings utilizados 10 o más veces en la indización de los artículos indizados en MEDLINE, sobre MP en HAD, ordenados según época de estudio

\begin{tabular}{|c|c|c|c|c|}
\hline \multirow[t]{2}{*}{$\begin{array}{l}\text { MeSH } \\
\text { Medical Subject Headings }\end{array}$} & \multicolumn{2}{|c|}{$\begin{array}{l}1^{\text {a época }} \\
1994 \text { a } 2003\end{array}$} & \multicolumn{2}{|c|}{$\begin{array}{l}2^{a} \text { época } \\
2003 \text { a } 2018\end{array}$} \\
\hline & núm. & $\%$ & núm. & $\%$ \\
\hline Home Care Services, Hospital-Based & 30 & 14,6 & 22 & 12,1 \\
\hline Home Infusion Therapy & 22 & 10,7 & 6 & 3,3 \\
\hline Antineoplastic Combined Chemotherapy Protocols & 16 & 7,8 & 12 & 6,6 \\
\hline Neoplasms & 9 & 4,4 & 11 & 6,0 \\
\hline Ambulatory Care & 6 & 2,9 & 14 & 7,7 \\
\hline Quality of Life & 3 & 1,5 & 12 & 6,6 \\
\hline Antineoplastic Agents & 7 & 3,4 & 6 & 3,3 \\
\hline Parenteral Nutrition, Home & 6 & 2,9 & 4 & 2,2 \\
\hline Colorectal Neoplasms & 5 & 2,4 & 5 & 2,7 \\
\hline
\end{tabular}

Se constataron diferencias estadísticamente significativas, relacionadas con los Descriptores MeSH utilizados, entre las dos épocas a estudio (chi cuadrado de Pearson =166,7; gl = 115; $p<$ $0,001)$.

Las frecuencias de las 14 grandes áreas temáticas donde se incluyeron los artículos indizados en la base de datos MEDLINE pueden consultarse en la tabla 5. No se observaron diferencias en su empleo entre las dos épocas analizadas (chi cuadrado de Pearson $=15,7 ; \mathrm{gl}=10 ; \mathrm{p}=0,11$ ).

\section{DISCUSIÓN}

Este estudio analiza los principales indicadores bibliométricos de la producción científica sobre la utilización de MP en HAD indizada en la base de datos bibliográfica MEDLINE, observando al mismo tiempo la clasificación derivada de los descriptores MeSH utilizados para su indización. Según el análisis efectuado, los documentos recuperados están en consonancia con la temática seleccionada.

La evolución de la producción científica mostró una tendencia exponencial decreciente, resultado opuesto a las teorías cienciométricas, las cuales determinan que tras períodos temporales entre 15 y 30 años se observa una "explosión de la información", en forma de crecimiento exponencial de 
la producción científica en una materia dada. Este hecho constata que el tema estudiado, a pesar de contar con más de 20 años desde las primeras publicaciones, nunca ha llegado a constituir un verdadero frente de investigación. Así, el año más productivo fue el 2006 y, a partir de él y contrariamente a lo esperado, se produjo un descenso en la generación de artículos. Quizá, este hecho se deba al enfoque altamente específico del análisis planteado (Ios MP) en un área temática (la HAD) que per se no ha alcanzado la "explosión de la información" (10).

En relación a la tipología documental observada, no es de extrañar que prácticamente el 50\% de los artículos sean originales al tratarse de un área de estudio con amplio campo de investigación asistencial. No obstante, y debido a la débil producción científica, el Índice de Productividad es inferior al observado en otros estudios bibliométricos de ciencias de la salud (11). Ahora bien, gracias al considerable número de ensayos clínicos, propio de un área plenamente clínica, el porcentaje de artículos citables es elevado, teniendo en cuenta que los indicadores de evaluación sugieren una proporción mínima del 50\% (12).

El análisis de la obsolescencia/actualidad, medido tanto por el Índice de Burton-Kleber como por el Índice de Price (1 de cada 10 artículos han sido publicados en los últimos 5 años), arrojó datos superiores a los previstos en el área de las ciencias de la salud (13) e incluso superiores a los obtenidos en el ámbito de la $\operatorname{HAD}(10,14)$, lo que reafirma el escaso interés que hasta el momento ha suscitado este tema.

Llama la atención el predominio del idioma japonés, con prácticamente la mitad de los artículos publicados en este idioma, circunstancia totalmente inusual en el ámbito de las ciencias de la salud, con predominio de publicaciones en lengua inglesa (8). La filiación mayoritariamente japonesa de los trabajos está íntimamente relacionada con el predominio de este idioma. En este sentido, cabe destacar que los resultados obtenidos se corresponden con los encontrados en otros estudios bibliométricos en el área de la HAD, en el que la procedencia geográfica, aunque no la mayoritaria, fue ampliamente japonesa (10). Este hecho podría explicarse por la extensa implantación de la asistencia domiciliaria en Japón, como consecuencia de la cronificación de los procesos médicos en el país con mayor longevidad del mundo.

Por otra parte, teniendo en cuenta que el inglés es el idioma aceptado por la mayoría de las revistas, no siendo así para otros idiomas (15), la predominante publicación en japonés penaliza su difusión, y presumiblemente ha lastrado el impacto de la materia estudiada.

No obstante, es importante destacar que en la segunda época, a pesar de que Japón continua siendo el principal país productor, el inglés se coloca como primer idioma de publicación. Esto es lógico, pues está demostrado que aquellos autores con una capacidad idiomática mayor, tienden a publicar en revistas de habla anglófona una vez realizado el esfuerzo de escribir el artículo. Además, pueden ser amparados por su propia institución que impulsa y recomienda el envío a revistas de alto impacto aunque ello suponga tener que pagar por la posterior recuperación del artículo o incluso por su revisión (16).

El indicador sobre colaboración institucional revela la no existencia de grandes grupos de investigación y la predominancia de pequeños grupos investigadores que trabajan de manera aislada con poca o nula colaboración con otros centros, con resultados inferiores a los publicados en otras áreas de ciencias de la salud (17). Estos resultados, pueden disminuir la visibilidad de la materia general estudiada y no contribuir a fomentar el desarrollo del conocimiento, sobre MP en HAD, como elemento central. Por estos motivos, quizás éste sea un punto clave a mejorar de cara al futuro de nuevos proyectos en esta línea investigadora (11).

El número de autores y el índice de cooperación presentaron datos superiores a los de otras revistas del ámbito de las ciencias de la salud $(8,11)$, aunque en concordancia con los resultados observados en estudios del ámbito de la HAD (14). A pesar de esto, es conveniente señalar que los datos analizados no mostraron inclinación hacia las autorías colectivas, lo cual indica una baja 
colaboración entre autores y en la formación de grupos de trabajo, elementos indispensables para el desarrollo de la ciencia. En todo caso, la leve evolución en el tiempo del número de autores por artículo es un dato alentador, aunque mejorable.

Sorprendente es el hecho de que una única revista, no específica en el tema, constituya el núcleo principal de Bradford, y más aún, una revista publicada en japonés. Este hecho está íntimamente ligado a la filiación predominantemente japonesa de los documentos estudiados. Según la Ley de Bradford, para cada área temática estudiada, sería suficiente identificar las "publicaciones núcleo" para esa área y este conjunto de publicaciones contendrán una tercera parte de todo lo publicado y generalmente los trabajos más visibles (18). El escaso impacto y posición de la revista que integra este núcleo principal de Bradford, denota la precaria difusión del conocimiento del tema estudiado.

El significativo incremento observado en el acceso al documento primario en la $2^{a}$ época es debido, obviamente, al desarrollo de las bases de datos bibliográficas, los buscadores propios de materia y las páginas web específicas de las revistas, y por supuesto, a la interconexión entre ellos. Queda demostrada la gran utilidad de Internet, ya que sin su existencia sería imposible disponer de este volumen de información y poder evaluarla. En cuanto al acceso al texto completo, de forma libre, permanente y gratuita, se comprueba el avance de la iniciativa Open Access acorde con los principales movimientos de apertura de la transmisión del conocimiento (19), aunque con un escaso apoyo (menos de 1 de cada 20 documentos analizados).

Es significativo la presencia, y aumento en el tiempo, de la utilización del Digital Object Identifier (DOI) ya que este identificador, similar a un identificador de recursos uniforme (URI), permite a los investigadores un acceso rápido y persistente al documento primario (20). Lo que es patente, es que su implantación y uso es, hoy por hoy, incuestionable. Por ende, que más de la mitad de los artículos estudiados en la segunda época tuviera DOI, facilita la recuperación de los artículos sobre $\mathrm{MP}$ en $\mathrm{HaD}$.

Cabe destacar que todos los datos referentes al acceso al documento primario son concordantes con estudios similares relacionados con el área temática (la HAD) (10).

El estudio y análisis de los descriptores y sus correspondientes áreas temáticas permitió conocer la clasificación de los documentos con relación a la temática a estudio (21). Se evidenció que los descriptores -MeSH- más utilizados y de mayor "peso" en las indizaciones de los artículos se adecuaban al tema de los MP en HAD (Home Care Services, Hospital-Based, Home Infusion Therapy, Antineoplastic Combined Chemotherapy Protocols.) y, asimismo, con el área temática que los jerarquiza (Health Care Category). No obstante, es importante resaltar la inexistencia de Descriptores (MeSH) específicos del concepto de «medicamento peligroso», lo que puede llevar, en el caso de búsquedas menos específicas que la que nos atañe, a la obtención de un gran ruido documental, como se observó en una revisión sistemática (5). La utilización, en ambas épocas de estudio, de los descriptores Antineoplastic Combined Chemotherapy Protocols y en menor medida, Neoplasms, revelan que la manipulación de MP en HAD se centra fundamentalmente en el grupo de los antineoplásicos.

Quizá, al reflexionar sobre los temas principales del jerárquico (las 16 principales categorías del thesaurus de la United States National Library of Medicin) puede extrañar la presencia de Psychiatry and Psychology Category así como Humanities Category, aunque ello es lógico si se tiene en cuenta que estos temas engloban descriptores como Attitude of Health Personnel, Patient Satisfaction y Attitude to Death, el primero, y Quality of Life, el segundo, todos ellos relacionados con la HAD, si se piensa en su amplio recorrido en la atención paliativa a pacientes, entre otros, con cáncer.

Como posible limitación de este estudio señalar que si bien se ha utilizado el autor designado para la correspondencia para estudiar la filiación institucional y esto puede ir en detrimento del resto de los autores del artículo, y ser posible sesgo, existen trabajos que han analizado la no existencia de diferencias significativas entre tomar sólo este autor o la totalidad de ellos $(8,10)$. Igualmente, 
se conoce que en MEDLINE existen limitaciones de campo, en las que hasta el año 2013, sólo se recogía la dirección del primer autor, hecho que conduce una restricción en la obtención de una mayor información (14).

\section{CONCLUSIONES}

Los estudios sobre MP en HAD son un área temática en decrecimiento, con una gran fragmentación y poco uniforme, sin grandes grupos de referencia ni una base sólida desde la que se puedan continuar estudios posteriores. El japonés es el idioma mayoritario. No obstante, la evolución del número de publicaciones en inglés en la segunda época de estudio es un dato alentador, aunque insuficiente. Los descriptores y las áreas temáticas son acordes al área estudiada, aunque sería conveniente, dada la creciente sensibilización con la manipulación de los MP, el desarrollo de un descriptor MeSH específico.

\section{BIBLIOGRAFÍA}

1. Minardi Mitre Cotta R, Morales Suárez-Varela M, Llopis González A, Cotta Filho JS, Real ER, Días Ricós JA. Home hospitalization: background, current situation, and future prospects. Rev Panam Salud Publica. 2001;10(1):45-55. PMID: 11558249

2. Massa Domínguez B. La hospitalización a domicilio en el siglo XXI. Hosp Domic. 2017;1(1):7-9. DOI: 10.22585/hospdomic.v1i1.8

3. González-Ramallo VJ, Segado-Soriano A. Twenty five years of hospital at home in Spain. Med Clin (Barc). 2006;126(9):332-3. PMID: 16650365

4. Joint Comission on Acreditation of Healthcare Organizations (JCAHO). Standards for the Accreditation of Home Care. Chicago, EEUU: JCAHO; 1988.

5. Bernabeu-Martínez MA, Ramos Merino M, Santos Gago JM, Álvarez Sabucedo LM, WandenBerghe C, Sanz-Valero J. Guidelines for safe handling of hazardous drugs: A systematic review. PLOS ONE. 2018;13(5):e0197172. DOI: 10.1371/journal.pone.0197172; PMID: 29750798

6. Sarewitz D. The pressure to publish pushes down quality. Nature. 2016;533(7602):147. DOI: 10.1038/533147a; PMID: 27172010

7. Castiel LD, Sanz-Valero J. Entre fetichismo e sobrevivência: o artigo científico é uma mercadoria acadêmica? Cad Saúde Pública. 2007;23(12):3041-50. DOI: 10.1590/S0102311X2007001200026; PMID: 18157347

8. Quesada-Risueño P, Sanz-Valero J, Wanden-Berghe C. Análisis bibliométrico de la producción científica existente en la base de datos bibliográfica MEDLINE sobre la fibra dietética. Rev Esp Nutr Humana Dietética. 2017;21(1):29. DOI: 10.14306/renhyd.21.1.275

9. Tsuji Y, Nakamura T, Katagiri E, Kawanishi J, Handa H, Honjo K, et al. Outpatient chemotherapy with continuous infusion of 5-fluorouracil (Cl 5-FU) and intravenous bolus leucovorin (IVB LV) in advanced gastrointestinal cancer. Gan To Kagaku Ryoho. 1994;21 Suppl 4:419-25. PMID: 7802444 
10. Sanz-Valero J, Wanden-Berghe C. Análisis bibliométrico de la producción científica, indizada en MEDLINE, sobre los servicios de salud proporcionados por las unidades de hospitalización a domicilio. Hosp Domic. 2017;1(1):21-34. DOI: 10.22585/hospdomic.v111.3

11. Palomo-Linares B, Palomo-Linares R, Sanz-Valero J. Análisis bibliométrico de los sistemas de medicación hospitalarios. Ars Pharm. 2017;58(1):29-37. DOI: 10.4321/s2340-98942017000100004

12. Sanz-Valero J, Casterá VT, Wanden-Berghe C. Bibliometric study of scientific output published by the Revista Panamericana de Salud Pública/Pan American Journal of Public Health from 1997-2012. Rev Panam Salud Publica. 2014;35(2):81-8. PMID: 24781088

13. Sanz-Valero J. Análisis bibliométrico y temático de la producción científica sobre ácidos grasos omega-3 indizada en las bases de datos internacionales sobre ciencias de la salud. Nutr Hosp. 2012;(2):51-58. DOI: 10.3305/nh.2012.27.sup2.6272; PMID: 23568396

14. Domingo-Pueyo A. Análisis bibliométrico de la producción científica española, indizada en MEDLINE, sobre servicios de atención a domicilio provisto por hospital. Hosp Domic. 2017;1(3):14151. DOI: 10.22585/hospdomic.v1i3.24

15. Franco-López A, Sanz-Valero J, Culebras JM. Publicar en castellano, o en cualquier otro idioma queno sea inglés, negativo para el factor de impacto. J Negat No Posit Results. 2106;1(2):6570. DOI: 10.19230/jonnpr.2016.1.2.1005

16. Sanz-Valero J. Estudio bibliométrico de producción y consumo de la revista Farmacia HospitaIaria (2004-2012). Farm Hosp. 2014;(1):1-8. DOI: 10.7399/FH.2014.38.1.1153 PMID: 24483853

17. Castiel LD, Sanz-Valero J. Política científica: manejar la precariedad de los excesos y desnaturalizar la idelología "publicionista" todopoderosa. Salud Colectiva. 2009;5(1):5-11.

18. National Institute of Standards and Technology. Bradford's law. In: Dictionary of Algorithms and Data Structures [Internet]. EEUU: U.S. National Institute of Standards and Technology; Available from: http://www.nist.gov/dads/

19. Abad-García MF, González-Teruel A, Argento J, Rodríguez-Gairín JM. Características y visibilidad de las revistas españolas en ciencias de la salud en bases de datos. El Prof Inf. 2015;24(5):537-50. DOI: 10.3145/epi.2015.sep.04

20. DeRisi S, Kennison R, Twyman N. The what and whys of DOls. PLoS Biol. 2003;1(2):e57. DOI: 10.1371/journal.pbio.0000057; PMID: 14624257

21. Palomo Llinares B, Sánchez Tormo J, Palomo Llinares R. Indización y uso de los Descriptores MeSH en Hospitalización a Domicilio. Hosp Domic. 2017;1(2):83-91. DOI: 10.22585/hospdomic. v1i2.5 